\title{
An in-depth study of a neutron star accreting at low Eddington rate: On the possibility of a truncated disk and an outflow
}

\author{
N. Degenaar ${ }^{1,2 \star}$, C. Pinto ${ }^{1}$, J.M. Miller ${ }^{3}$, R. Wijnands ${ }^{2}$, D. Altamirano ${ }^{4}$, F. Paerels ${ }^{5,6}$, \\ A.C. Fabian ${ }^{1}$ and D. Chakrabarty ${ }^{7}$ \\ ${ }^{1}$ Institute of Astronomy, University of Cambridge, Madingley Road, Cambridge CB3 OHA, UK \\ ${ }^{2}$ Anton Pannekoek Institute for Astronomy, University of Amsterdam, Science Park 904, 1098 XH, Amsterdam, the Netherlands \\ ${ }^{3}$ Department of Astronomy, University of Michigan, 1085 South University Avenue, Ann Arbor, MI 48109, USA \\ ${ }^{4}$ Department of Physics and Astronomy, University of Southampton, Southampton, Hampshire, SO171BJ, UK \\ ${ }^{5}$ Columbia University, Mail Code 5246, 550 West 120th Street, New York, NY 10027, USA \\ ${ }^{6}$ Columbia Astrophysics Laboratory, Mail Code 5247, 550 West 120th Street, New York, NY 10027, USA \\ ${ }^{7}$ Massachusetts Institute of Technology (MIT), Kavli Institute for Astrophysics and Space Research, Cambridge, MA 02139, USA
}

Accepted 2016 September 12. Received 2016 September 12; in original form 2016 August 16

\begin{abstract}
Due to observational challenges our knowledge of low-level accretion flows around neutron stars is limited. We present NuSTAR, Swift and Chandra observations of the low-mass Xray binary IGR J17062-6143, which has been persistently accreting at $\simeq 0.1$ per cent of the Eddington limit since 2006. Our simultaneous NuSTAR/Swift observations show that the 0.5$79 \mathrm{keV}$ spectrum can be described by a combination of a power law with a photon index of $\Gamma \simeq 2$, a black body with a temperature of $k T_{\mathrm{bb}} \simeq 0.5 \mathrm{keV}$ (presumably arising from the neutron star surface), and disk reflection. Modeling the reflection spectrum suggests that the inner accretion disk was located at $R_{\text {in }} \gtrsim 100 G M / c^{2}(\gtrsim 225 \mathrm{~km})$ from the neutron star. The apparent truncation may be due to evaporation of the inner disk into a radiatively-inefficient accretion flow, or due to the pressure of the neutron star magnetic field. Our Chandra gratings data reveal possible narrow emission lines near $1 \mathrm{keV}$ that can be modeled as reflection or collisionally-ionized gas, and possible low-energy absorption features that could point to the presence of an outflow. We consider a scenario in which this neutron star has been able to sustain its low accretion rate through magnetic inhibition of the accretion flow, which gives some constraints on its magnetic field strength and spin period. In this configuration, IGR J17062-6143 could exhibit a strong radio jet as well as a (propeller-driven) wind-like outflow.
\end{abstract}

Key words: accretion: accretion disks - pulsars: general - stars: individual (IGR J170626143 ) - stars: neutron - X-rays: binaries - X-rays: bursts

\section{INTRODUCTION}

Low-mass X-ray binaries (LMXBs) contain a neutron star or a black hole that accretes gas from a less massive companion star. These are excellent laboratories to study accretion in the strong gravity regime. In particular, LMXBs are observed over a wide range in X-ray luminosity, hence accretion rate, allowing the investigation of different accretion morphologies.

LMXBs are most easily discovered and studied when their $\mathrm{X}$-ray luminosity is a sizable fraction of the Eddington limit, $L_{\mathrm{X}} \gtrsim 0.1 L_{\mathrm{Edd}}$. However, a lot of accretion activity occurs at much lower $L_{\mathrm{X}}$. Indeed, many LMXBs are transient and accrete at $L_{\mathrm{X}}>0.1 L_{\mathrm{Edd}}$ for only a few weeks or months and then spend years in quiescence before a new outburst commences. Thermalviscous instabilities in the accretion disk provide the general frame-

* e-mail: degenaar@ast.cam.ac.uk work for understanding such outburst-quiescence cycles (though it cannot explain details; e.g. Lasota 2001, for a review).

Accretion does not necessarily switch off in quiescence and may persist down to very low $L_{\mathrm{X}}$ (e.g. Wagner et al. 1994; Campana et al. 1997; Rutledge et al. 2002; Kuulkers et al. 2009; Cackett et al. 2010a, 2013; Bernardini et al. 2013; Chakrabarty et al. 2014; D’Angelo et al. 2015; Rana et al. 2016). Furthermore, there is a growing population of LMXBs that exhibit outbursts with a peak luminosity of only $L_{\mathrm{X}} \simeq 10^{-4}-10^{-2} L_{\mathrm{Edd}}$ (e.g. Hands et al. 2004; Sakano et al. 2005; Muno et al. 2005; Wijnands et al. 2006; Degenaar \& Wijnands 2009; Campana 2009; Heinke et al. 2010; Armas Padilla et al. 2011, 2014; Sidoli et al. 2011). Understanding the properties of LMXBs at $L_{\mathrm{X}}<10^{-2} L_{\mathrm{Edd}}$ is thus an important part of forming a complete picture of accretion flows around neutron stars and stellar-mass black holes.

At $L_{\mathrm{X}}>10^{-2} L_{\mathrm{Edd}}$, matter is typically transferred through a geometrically thin and optically thick accretion disk that ex- 
tends close to the compact primary. When moving towards lower $L_{\mathrm{X}}$, the inner disk is expected to evaporate into a hot, geometrically thick and radiatively inefficient accretion flow (e.g. Narayan \& Yi 1994; Blandford \& Begelman 1999; Menou et al. 2000; Dubus et al. 2001). The X-ray spectral softening observed as black hole LMXBs transition from outburst to quiescence seems to support the formation of a radiatively-inefficient accretion flow (see e.g. Plotkin et al. 2013; Reynolds et al. 2014; Yang et al. 2015, for discussions).

Neutron star LMXBs also show X-ray spectral softening at $L_{\mathrm{X}}<10^{-2} L_{\mathrm{Edd}}$ (e.g. Armas Padilla et al. 2011, 2013b; Degenaar et al. 2013b; Bahramian et al. 2014; Allen et al. 2015; Weng et al. 2015), although the behavior is not the same as for black holes (Wijnands et al. 2015). The X-ray spectra are also different; high-quality data obtained for neutron stars at $L_{\mathrm{X}} \simeq 10^{-4} L_{\mathrm{Edd}}$ have revealed the presence of a thermal component, likely from the accretion-heated neutron star surface, and a power-law spectral component that is harder than for black holes at similar $L_{\mathrm{X}}$ (e.g. Armas Padilla et al. 2013a b; Degenaar et al. 2013b; Wijnands et al. 2015). The picture proposed for neutron star LMXBs is that the radiation from the accretion flow softens at $L_{\mathrm{X}}<10^{-2} L_{\text {Edd }}$ like in black holes, but becomes overwhelmed by the emission released when matter impacts the neutron star surface (causing thermal emission and a hard tail) at $L_{\mathrm{X}}<10^{-3} L_{\mathrm{Edd}}$ (Wijnands et al. 2015). Deep observations of the nearby neutron star Cen X-4 at $L_{\mathrm{X}} \simeq 4 \times 10^{-6} L_{\mathrm{Edd}}$ may support this idea; it appears that only thermal emission from the stellar surface and bremsstrahlung from a boundary layer (where the accretion flow meets the surface) are observed, whereas the accretion flow itself is not directly detected (Chakrabarty et al. 2014; D'Angelo et al. 2015).

The magnetic field of a neutron star may also have a discernible effect on the accretion flow. It can potentially truncate the inner disk and re-direct plasma along the magnetic field lines. X-ray pulsations from the heated magnetic poles may then be seen (e.g. Pringle \& Rees 1972; Rappaport et al. 1977; Finger et al. 1996; Wiinands \& van der Klis 1998). X-ray spectral observations revealed truncated inner disks in several X-ray pulsars (e.g. Miller et al. 2011; Papitto et al. 2013; Degenaar et al. 2014a; King et al.2016; Pintore et al.2016), whereas the inner disk seems to extend further in for non-pulsating neutron star LMXBs (e.g. Cackett et al. 2010b; Miller et al. 2013; Degenaar et al. 2015; Di Salvo et al. 2015; Ludlam et al. 2016). Magnetic field effects can possibly gain importance when the accretion rate drops, allowing the magnetic pressure to increasingly compete with that exerted by the disk.

Since transient LMXBs typically only spend a short time at $L_{\mathrm{X}} \simeq 10^{-4}-10^{-2} L_{\mathrm{Edd}}$, this is a particularly challenging accretion regime to characterize and to capture with sensitive observations. Fortuitously, a handful of neutron star LMXBs accrete in this range for several years (e.g. Chelovekov \& Grebenev 2007; Del Santo et al. 2007; Jonker \& Keek 2008; Heinke et al. 2009; in 't Zand et al. 2009; Degenaar et al. 2010, 2012b; Armas Padilla et al. 2013a). These very-faint X-ray binaries (VFXBs) are interesting targets to further our knowledge of low-level accretion flows.

VFXBs are also intriguing because the disk instability model has trouble explaining how their low accretion rates can be sustained for many years (e.g. Dubus et al. 1999; Lasota 2001). One possibility is that these objects have small binary orbits that only fit small accretion disks (e.g. King \& Wijnands 2006; in 't Zand et al. 2007; Hameury \& Lasota 2016). The very dim optical counterparts indeed suggests that some VFXBs may have short orbital periods (e.g. Bassa et al.2008; in 't Zand et al.2009). However, a few other VFXBs were found to harbor H-rich donors, which rules out very compact orbits (e.g. Degenaar et al. 2010; Arnason et al. 2015). An alternative explanation for the quasi-stable low accretion rate of VFXBs is that the neutron star's magnetic field inhibits the accretion flow (e.g. Wiinands 2008; Heinke et al. 2009, 2015; Patruno 2010a; Degenaar et al. 2014b).

\subsection{The very-faint X-ray binary IGR J17062-6143}

IGR J17062-6143 was discovered with Integral in 2006 (Churazov et al. 2007), but it was not until 2012 that it was identified as a neutron star LMXB through the detection of an energetic thermonuclear X-ray burst (Degenaar et al.2012a). The X-ray burst light curve showed wild intensity variations that were presumably caused by extreme expansion of the neutron star photosphere; this suggests a source distance of $D \simeq 5 \mathrm{kpc}$ (Degenaar et al. 2013a), assuming that the peak flux of the X-ray burst reached the empirical Eddington limit of $L_{\text {Edd }}=3.8 \times 10^{38} \mathrm{erg} \mathrm{s}^{-1}$ (Kuulkers et al. 2003). The source has been persistently accreting at a low luminosity of $L_{X} \simeq 4 \times 10^{35}(D / 5.0 \mathrm{kpc})^{2} \mathrm{erg} \mathrm{s}^{-1}$ for the past $10 \mathrm{yr}$, which roughly corresponds to $\simeq 10^{-3} L_{\mathrm{Edd}}$ (e.g. Ricci et al. 2008; Remillard \& Levine 2008; Degenaar et al. 2012a). It was not detected by the ROSAT/PSPC in 1990 (obsID rs932824n00), suggesting that its luminosity was likely a factor $\gtrsim 10$ lower at that time.

Due to its relative proximity and relatively low interstellar extinction compared to other VFXBs, IGR J17062-6143 is a particularly good target to further our understanding of low-level accretion flows and the nature of these peculiar LMXBs. In particular, the powerful X-ray burst seen in 2012 revealed the presence of $\mathrm{Fe}$ in the accreted matter (Degenaar et al. 2013a), which provides the chance of detecting reflection features from the accretion disk.

Disk reflection manifests itself most prominently as an $\mathrm{Fe}-\mathrm{K}$ emission line at $\simeq 6.4-6.97 \mathrm{keV}$ and a Compton hump at $\simeq 20-40$ keV (e.g. George \& Fabian 1991; Matt et al. 1991). The shape of these features is modified by Doppler and gravitational redshift effects as the gas in the disk moves in high-velocity Keplerian orbits inside the gravitational well of the compact accretor. The reflection spectrum thus encodes information about the accretion morphology (e.g. Fabian \& Ross 2010, for a review). In particular, detecting and modeling disk reflection features allows for a measure of the inner radial extent of the accretion disk, $R_{\text {in }}$. If a radiatively-inefficient accretion flow forms or if the stellar magnetic field is dynamically important in governing the accretion flow in VFXBs, the inner disk is expected to be truncated away from the neutron star. However, so far no observational constraints on the inner radial extent of the accretion disks in VFXBs have been obtained yet.

Radiatively-inefficient accretion flows are likely associated with outflows (e.g. Rees et al. 1982; Narayan \& Yi 1994; Blandford \& Begelman 1999; Narayan 2005). Furthermore, the magnetic field of a neutron star may act as a propeller and could expel (some of) the in-falling gas (e.g. Illarionov \& Sunyaev 1975; Lovelace et al. 1999; Romanova et al. 2009; Papitto \& Torres 2015). Neutron star LMXBs accreting at $L_{\mathrm{X}}<10^{-2} L_{\mathrm{Edd}}$ may therefore be expected to exhibit outflows. In the X-ray band, these may reveal itself through the detection of blue-shifted narrow spectral lines.

In this work we present NuSTAR, Swift and Chandra/HETG observations of IGR J17062-6143 to study the accretion regime of $L_{\mathrm{X}} \simeq 10^{-3} L_{\mathrm{Edd}}$ and to understand the puzzling nature of neutron star LMXBs that are able to accrete at such a low rate for years. In 
particular, the aim of these observations was to constrain the continuum spectral shape, to measure disk reflection features to gain insight into the accretion geometry, and to search for narrow X-ray spectral lines that may be indicative of an outflow.

\section{OBSERVATIONS AND DATA ANALYSIS}

\subsection{NuSTAR}

Our NUSTAR observation was performed between 19:26 UT on 2015 May 6 and 05:01 UT on May 8 (obs ID 30101034002). The two co-aligned focal plane modules (FPM) A and B provide an energy coverage of 3-79 $\mathrm{keV}$. Standard processing with NUSTARDAS (v. 1.4.1), resulted in $\simeq 70 \mathrm{ks}$ of on-target exposure time per module. We extracted light curves, spectra, and response files with NUPRODUCTS, using a circular region of $30^{\prime \prime}$ radius for the source and a void circular region of $60^{\prime \prime}$ radius on the same chip for the background. IGR J17062-6143 was detected at a constant intensity of $\simeq 3 \mathrm{c} \mathrm{s}^{-1}$ during the observation (3-79 keV, FPMA+B summed).

When fitting the FPMA/B spectra simultaneously with a constant factor floating in between, we found that the flux calibration agreed to within $0.5 \%$. We therefore opted to combine the spectra of the two mirrors using ADDASCASPEC. A weighted response file was created using ADDRMF. The combined spectrum was grouped into bins with a minimum of 20 photons using GRPPHA. In the combined spectrum the source was detected above the background in the entire NUSTAR bandpass; the signal to noise ratio (SNR) was $\simeq 500$ around $7 \mathrm{keV}$ and $\simeq 2$ around $70 \mathrm{keV}$.

\subsection{Swift/XRT}

Swift observed IGR J17062-6143 simultaneous with NuSTAR on 2015 May 6 from 23:29-23:44 UT (obs ID 37808005). A $\simeq 0.9 \mathrm{ks}$ exposure was obtained during a single orbit and the XRT was operated in photon counting mode. The source was detected at a constant count rate of $\simeq 0.9 \mathrm{c} \mathrm{s}^{-1}$. Source and background spectra were extracted using XSELECT. To circumvent the effects of pile-up, we used an annular region with inner-outer radii of $12^{\prime \prime}-71^{\prime \prime}$ for the source. Background events were extracted from a void region with an area three times larger than that used for the source. An arf was created with XRTMKARF, using the exposuremap as input. The appropriate rmf (v. 15) was taken from the CALDB. The spectral data were grouped into bins with a minimum of 20 photons.

\subsection{Chandra/HETG}

We observed IGR J17062-6143 with Chandra on 2014 October 25 from 04:48 to 13:39 UT for $\simeq 29 \mathrm{ks}$ of on-source exposure (obs ID 15749), and for $\simeq 64 \mathrm{ks}$ from 09:34 UT on October 27 to $14: 57$ UT on October 28 (obs ID 17543). The High Energy Transmission Gratings (HETG) were used to disperse the incoming light on to the ACIS-S CCDs that were operated in faint, timed mode. The HETG consists of the Medium Energy Grating (MEG; 0.4$5 \mathrm{keV}, 31-2.5 \AA$ ) and the High Energy Grating (HEG; 0.8-8 keV, 15-1.2 $\AA$ ). We used the data from both grating arms.

The data were reprocessed using the CHAN_REPRO script. We extracted light curves for the first orders of the HEG and MEG instruments at 100-s resolution using the task DMEXTRACT. IGR J17062-6143 was detected at similar, constant count rates of $\simeq 1.9 \mathrm{c} \mathrm{s}^{-1}$ during the two observations (MEG+HEG combined; $0.4-8 \mathrm{keV})$. We extracted the first order HEG and MEG spectra from the reprocessed pha2 files employing DMTYPE2SPLIT, and generated response files using MKTGRESP.

We used COMBINE_GRATING_SPECTRA to combine the spectra of the plus and minus orders of each instrument, and to sum the data from the two observations. To investigate the spectral continuum shape and flux, we grouped the combined spectra to a minimum of 20 photons bin $^{-1}$. To search for narrow spectral features, we instead fitted the un-binned spectra of the plus and minus orders separately. A constant multiplication factor was always included in the modeling to allow for calibration differences.

\subsection{Spectral analysis}

For the analysis of the continuum and reflection spectrum, we used XSPEC (v. 2.9 Arnaud 1996) and applied $\chi^{2}$-statistics. Interstellar absorption was included in all our spectral fits using the TBABS model, employing cross-sections from Verner et al. (1996) and abundances from Wilms et al. (2000). For our high-resolution spectral analysis of the Chandra data, we instead employed SPEX (v. 3.0 1 because it is more suitable for finding and modeling narrow spectral features than XSPEC. Throughout this work we assume a source distance of $D=5 \mathrm{kpc}$ (Degenaar et al. 2013a), an Eddington luminosity of $L_{\mathrm{Edd}}=3.8 \times 10^{38} \mathrm{erg} \mathrm{s}^{-1}$ (Kuulkers et al. 2003), and a neutron star mass and radius of $M=1.4 \mathrm{M}_{\odot}$ and $R=10 \mathrm{~km}$, respectively. All errors are given at $1 \sigma$ confidence level.

\section{RESULTS}

\subsection{NuSTAR/Swift continuum X-ray spectrum}

A single power-law model (PEGPWRLW) does not provide a good fit to the $0.5-79 \mathrm{keV} \mathrm{NuSTAR/Swift}$ spectrum $\left(\chi_{\nu}^{2}=1.52\right.$ for 583 d.o.f.). Adding a soft, thermal component (BBODYRAD) yields a significant improvement $\left(\chi_{\nu}^{2}=1.15\right.$ for 581 d.o.f.; $F$-test probability $\simeq 10^{-36}$ ). We obtain $N_{\mathrm{H}}=(2.3 \pm 0.4) \times 10^{21} \mathrm{~cm}^{-2}$ (comparable to that found in previous studies; e.g. Ricci et al. 2008; Degenaar et al.2013a) $, \Gamma=2.10 \pm 0.01, k T_{\mathrm{bb}}=0.46 \pm 0.03 \mathrm{keV}$ and $R_{\mathrm{bb}}=3.8 \pm 0.4(\mathrm{~km} / 5.0 \mathrm{kpc})^{2}$. Replacing the power-law component by a Comptonized emission component (NTHCOMP) yielded a worse fit $\left(\chi_{\nu}^{2}=1.21,580\right.$ d.o.f.). We also tried cutoff and broken power-law models, but these revealed no evidence for a detectable change in the power-law index in the $0.5-79 \mathrm{keV}$ spectrum.

Our PEGPWRLW+BBODYRAD continuum fit is shown in Figure 1 (top). The inferred $0.5-79 \mathrm{keV}$ unabsorbed flux is $F_{0.5-79}=(1.17 \pm 0.02) \times 10^{-10} \mathrm{erg} \mathrm{cm}^{-2} \mathrm{~s}^{-1}$, which corresponds to $L_{0.5-79}=(3.50 \pm 0.06) \times 10^{35}(D / 5.0 \mathrm{kpc})^{2} \mathrm{erg} \mathrm{s}^{-1}$. This suggests that IGR J17062-6143 was accreting at $\simeq 10^{-3} L_{\mathrm{Edd}}$ during our $2015 \mathrm{NuSTAR/Swift}$ observations. The spectrum in the NUSTAR band is dominated by the power-law spectral component, which contributes $\simeq 97 \%$ to the total unabsorbed $3-79 \mathrm{keV}$ flux. For reference, the $0.5-10 \mathrm{keV}$ luminosity inferred from this fit is $L_{0.5-10} \simeq 1.6 \times 10^{35}(D / 5.0 \mathrm{kpc})^{2} \mathrm{erg} \mathrm{s}^{-1}$. In this energy band the power-law spectral component also dominates, accounting for $\simeq 79 \%$ of the total unabsorbed flux.

Our continuum description leaves positive residuals near $\simeq 6$ $7 \mathrm{keV}$, as shown in Figure 2 The feature is reminiscent of the broad $\mathrm{Fe}-\mathrm{K}$ lines that are often seen in the X-ray spectra of bright LMXBs

1 https://www.sron.nl/spex 

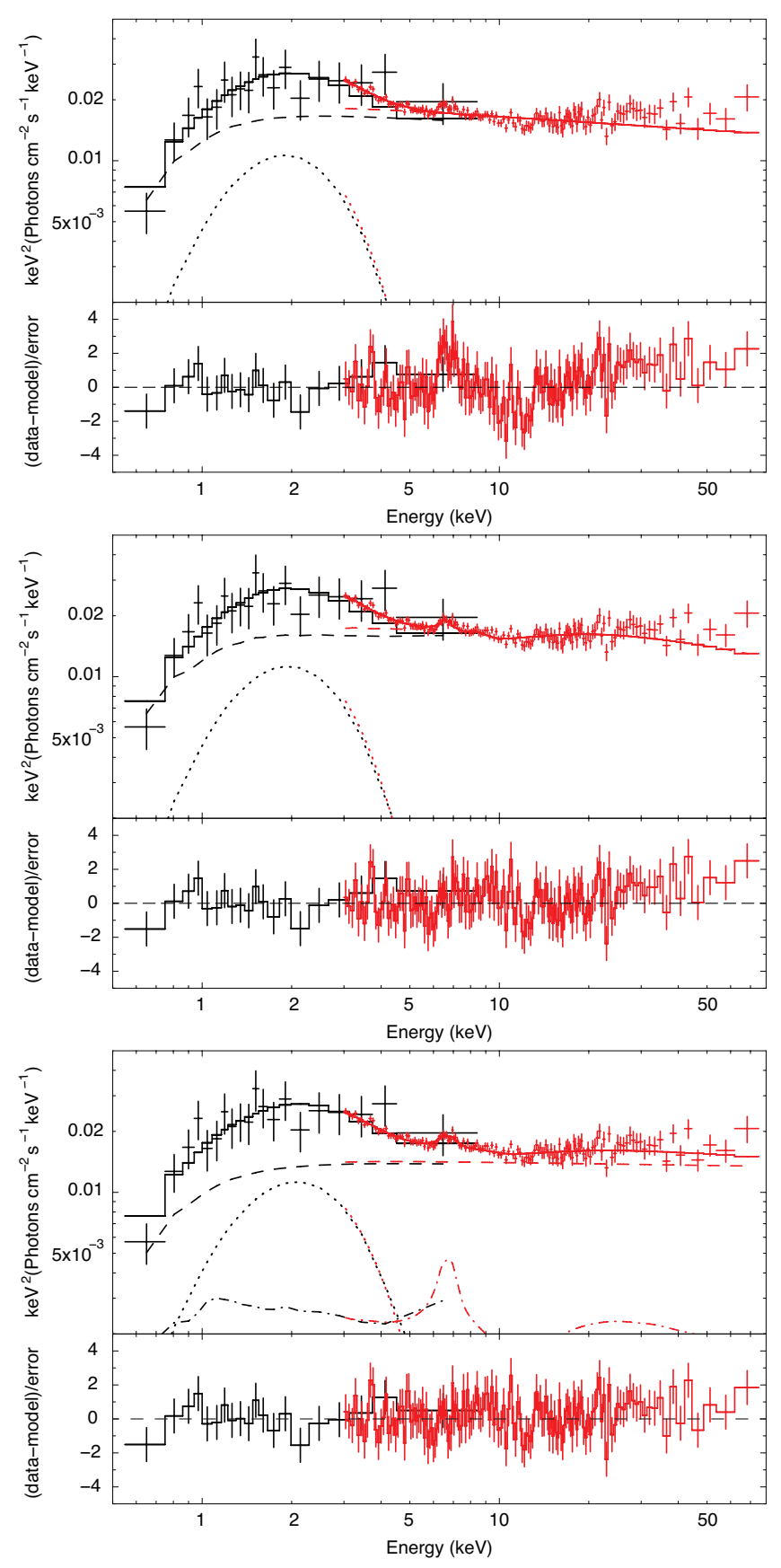

Figure 1. Fits to the NUSTAR (red) and Swift (black) spectral data. The lower panels show the residuals in sigmas. Top: Continuum fit to an absorbed black body (dotted curve) and power law (dashed curve) model. Middle: Fit with the power-law component replaced by RELXILL to include relativistic reflection (dashed curve). Bottom: Fit with a black body (dotted curve) plus power law (dashed curve) continuum and REFLIONX (dasheddotted curve) as the relativistic reflection model. We note that the highest NuSTAR and lowest Swift energy bins have a low SNR.

and are typically attributed to disk reflection 2 This motivates the inclusion of a relativistic reflection model in our spectral fits.

${ }^{2}$ See e.g. Ng et al. (2010) for a discussion on alternative explanations, but see Chiang et al. (2016) for a strong test case that favors disk reflection.

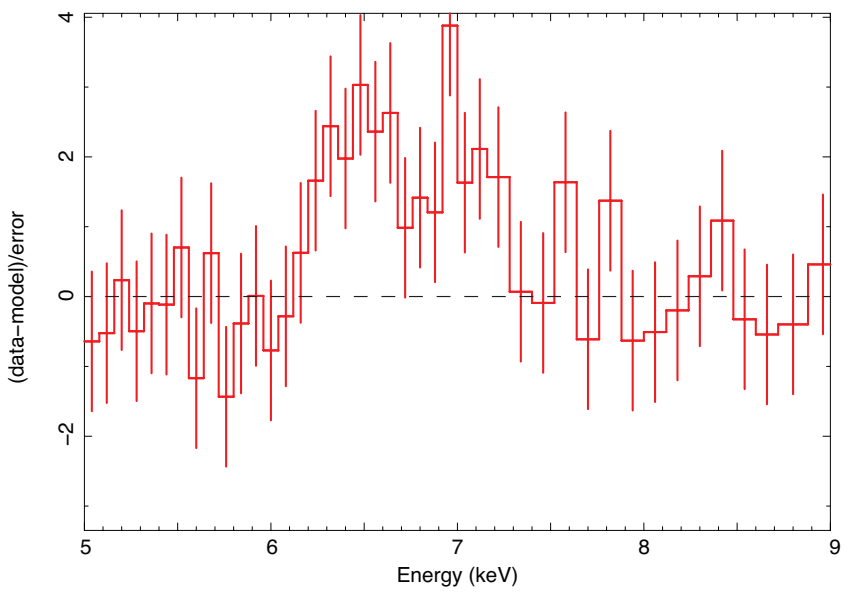

Figure 2. Zoom of the NUSTAR data to model ratio in the Fe-K line region for an absorbed power law plus black body continuum model.

\subsection{NuSTAR/Swift X-ray reflection spectrum}

\subsubsection{Reflection fits with RELXILL}

The shape of the reflection spectrum depends on the properties of the flux incident on the accretion disk. In case of IGR J170626143 , the $X$-ray spectrum is dominated by a $\Gamma \simeq 2$ power-law component (Figure 1 top). Out of all the reflection models appropriate for an incident power-law spectrum, we chose RELXILL (v. 0.4a; García et al. 2014), because it features higher spectral resolution and updated atomic data compared to other models. Furthermore, the reflection spectrum is calculated for each emission angle, which should be more accurate than averaging over different angles. The model combines the reflection grid XILLVER (García et al. 2013) with the convolution kernel RELCONV to include relativistic effects on the shape of the reflection spectrum (Dauser et al. 2010). Since it describes both the illuminating power-law and the reflected emission, we replaced PEGPWRLW by RELXILL in our spectral fits.

We set the model up using an unbroken emissivity profile of the form $\epsilon(r) \propto r^{-q}$. Reflection fits for LMXBs are typically not sensitive to the outer disk radius because the emissivity profile drops off steeply with increasing radius, so we fixed $R_{\text {out }}=500 R_{\mathrm{ISCO}}$, where $R_{\mathrm{ISCO}}$ is the location of the innermost stable circular orbit (ISCO). The remaining model parameters are then the index $\Gamma$ and the high-energy cutoff $E_{\text {cut }}$ of the illuminating power law, the dimensionless spin parameter $a$, the disk inclination $i$, the inner disc radius $R_{\text {in }}$ (expressed in terms of $R_{\mathrm{ISCO}}$ ), the ionization parameter $\log \xi$, the iron abundance $A_{\mathrm{Fe}}$ (with respect to Solar), the reflection fraction $R_{\text {refl }}$, and the normalization $N_{\text {refl }}$. We note that figure 1 of Fabian et al. (1989) gives an instructive overview of the effect of several of these parameters on the shape of relativistically-broadened Fe-K lines.

The spin of the compact object plays a role in setting $R_{\mathrm{ISCO}}$, but for neutron stars this is sufficiently low $(a \simeq 0-0.3)$ to be only a small effect (e.g. Miller et al. 2013). The spin of IGR J17062-6143 is unknown, but neutron stars in LMXBs are expected to be spun up to millisecond periods due to the angular momentum gained by accretion (e.g. Alpar et al. 1982; Bhattacharya \& van den Heuvel 1991; Strohmayer et al. 1996; Wijnands \& van der Klis 1998). This is indeed borne out by observations; spin periods of $\simeq 1.6-10 \mathrm{~ms}$ have been inferred for about two dozen neutron star LMXBs from detecting coherent X-ray pulsations or rapid intensity oscillations during thermonuclear X-ray bursts (e.g. Patruno 2010b, 
for a list). Here, we assumed $a=0.3$ based on the approximation $a \simeq 0.47 / P_{\mathrm{ms}}$, where $P_{\mathrm{ms}}$ is the spin period in ms (valid for $M=1.4 \mathrm{M}_{\odot} ;$ Braje et al. 2000). According to equation 3 from Miller et al. (1998), the ISCO is then located at $R_{\mathrm{ISCO}} \simeq 6[1-$ $0.54 a] G M / c^{2} \simeq 5.05 G M / c^{2}$, which corresponds to $\simeq 11.2 \mathrm{~km}$ for $M=1.4 \mathrm{M}_{\odot}$. Indeed, setting $a=0$ (i.e. the Schwarzschild metric with $R_{\mathrm{ISCO}}=6 \mathrm{GM} / \mathrm{c}^{2}$ ) did not strongly affect our inferred inner disk radius (see below).

Several model parameters were not constrained by the data when left free to vary and were therefore fixed to avoid degeneracy. Firstly, we set $q=3$, which is theoretically motivated (e.g. Wilkins \& Fabian 2012) and often applies well to neutron star LMXBs (e.g. Cackett et al. 2010b, for a sample study). Secondly, $E_{\text {cut }}$ pegged at the model upper limit of $1 \mathrm{MeV}$. Since our continuum modeling showed no evidence for an observable cutoff in the NUSTAR passband we fixed $E_{\text {cut }}=500 \mathrm{keV}$. This is a somewhat arbitrarily high value but consistent with that found from $\mathrm{NuS}$ TAR reflection modeling of the neutron star LMXB 4U 1608-52 at $L_{\mathrm{X}} \simeq 10^{-2} L_{\text {Edd }}$ (Degenaar et al.2015). We later tested how different values of $E_{\text {cut }}$ affected our fits (see below). We also found the disk inclination to be unconstrained, pegging at the upper limit $90^{\circ}$. Such a high inclination is ruled out by the fact that IGR J170626143 does not show dips or eclipses in its X-ray emission, which suggests $i \lesssim 75^{\circ}$ (e.g. Frank et al. 2002). As there is no typical value for the inclination of neutron star LMXBs, we therefore explored fits with $i=25^{\circ}, 45^{\circ}$, and $65^{\circ}$. Finally, we fixed $A_{\mathrm{Fe}}=1$ because it was poorly constrained when left free to vary. The only free fit parameters for RELXILL thus were $R_{\text {in }}, \Gamma, \log \xi, R_{\text {refl }}$, and $N_{\text {refl. }}$.

Our spectral analysis results are summarized in Table $1 \mathrm{Re}-$ placing the power law by RELXILL provides a significant improvement. The statistical quality of the fit with $i=65^{\circ}\left(\chi_{\nu}^{2}=1.01\right.$ for 578 d.o.f.) is better than the fits with $i=25^{\circ}$ and $45^{\circ}$ ( $\chi_{\nu}^{2}=1.02$ for 578 d.o.f.) at $\mathrm{a} \simeq 2-2.5 \sigma$ level $\left(\Delta \chi^{2}=4.31-6.34\right)$. The effect of increasing the inclination is to shift the blue wing of the Fe-K line to higher energy (see e.g. figure 1 of Fabian et al. 1989). The blue wing of the broad line in IGR J17062-6143 extends all the way up to $\simeq 7.3 \mathrm{keV}$ (Figure 2), which may be reason that a higher inclination is preferred in our reflection fits. We opted to proceed our analysis with $i=65^{\circ}$, but note that our conclusions are not affected by this choice. Our baseline fit described above is shown in Figure 1 (middle). We note that the highest NuSTAR and lowest Swift energy bins deviate from the model fit. This is plausibly due to a low SNR, but we explore the possible presence of an additional emission component in Section 3.3

For $i=65^{\circ}$, we obtained $R_{\text {in }}=44.4_{-24.6}^{*} R_{\mathrm{ISCO}}$ with the upper bound hitting the model limit of $100 R_{\mathrm{ISCO}}$. This inferred inner disk radius corresponds to $\simeq 224_{-125}^{*} G M / c^{2}$ for our choice of $a=0.3$, or $\simeq 497_{-278}^{*} \mathrm{~km}$ for a neutron star mass of $M=1.4 \mathrm{M}_{\odot}$. Notably, the different inclinations yield comparable $1 \sigma$ lower limits of $R_{\text {in }} \gtrsim 20 R_{\mathrm{ISCO}}\left(\gtrsim 100 \mathrm{GM} / \mathrm{c}^{2}\right.$ ), suggesting a truncated disk regardless of the chosen $i$. However, we note that the $\chi^{2}$-space is rather flat and $R_{\text {in }}$ is still consistent with a location at the ISCO at $\simeq 3 \sigma$ confidence for our fits with $i=65^{\circ}$.

We explored the effect of our model assumptions for the obtained value of $R_{\text {in }}$, our main parameter of interest. Table 2 summarizes the effect of changing the values of fixed model parameters ( $a, q, E_{\text {cut }}, A_{\mathrm{Fe}}$, and $\left.R_{\text {out }}\right)$. This shows that the $1 \sigma$ lower limit on $R_{\text {in }}$ always lies near $\simeq 20 R_{\text {ISCO }}$. A high cutoff energy appears to be preferred by our fits, which is consistent with the lack of an observable high-energy roll-over in the NUSTAR data. The reflection fits also appear to favor a high Fe abundance; this enhances the strength of the Fe-K line (e.g. Ross \& Fabian 2005).
Table 1. Results from modeling the NUSTAR/Swift reflection spectrum.

\begin{tabular}{lccc}
\hline Model parameter & \multicolumn{3}{c}{ RELXILL } \\
\hline & $i=25^{\circ}$ & $i=45^{\circ}$ & $i=65^{\circ}$ \\
$C(\mathrm{XRT})$ & $0.92 \pm 0.05$ & $0.92 \pm 0.08$ & $0.92 \pm 0.08$ \\
$N_{\mathrm{H}}\left(\times 10^{21} \mathrm{~cm}^{-2}\right)$ & $2.43 \pm 0.26$ & $2.47 \pm 0.35$ & $2.42 \pm 0.36$ \\
$k T_{\mathrm{bb}}(\mathrm{keV})$ & $0.48 \pm 0.02$ & $0.47 \pm 0.02$ & $0.48 \pm 0.02$ \\
$N_{\mathrm{bb}}(\mathrm{km} / 10 \mathrm{kpc})^{2}$ & $52.1_{-12.0}^{+2.0}$ & $53.5_{-12.4}^{+20.5}$ & $50.8_{-13.5}^{+9.9}$ \\
$\Gamma$ & $2.05 \pm 0.01$ & $2.05 \pm 0.02$ & $2.05 \pm 0.02$ \\
$R_{\text {in }}\left(R_{\mathrm{ISCO}}\right)$ & $97.6_{-61.3}^{*}$ & $98.1_{-78.9}^{*}$ & $44.4_{-24.6}^{*}$ \\
$\log \xi$ & $3.28 \pm 0.04$ & $3.27 \pm 0.08$ & $3.19 \pm 0.11$ \\
$R_{\text {refl }}$ & $0.24 \pm 0.03$ & $0.47 \pm 0.05$ & $0.48 \pm 0.09$ \\
$N_{\text {reff }}\left(\times 10^{-4}\right)$ & $2.41 \pm 0.45$ & $2.37 \pm 0.10$ & $2.29 \pm 0.11$ \\
$\chi_{\nu}^{2}($ dof $)$ & $1.02(578)$ & $1.02(578)$ & $1.01(578)$ \\
\hline
\end{tabular}

Notes. - The constant factor $C$ was set to 1 for NuSTAR and left free for the Swift data. An asterisk indicates that a parameter pegged at the model limit. Quoted errors reflect $1 \sigma$ confidence levels.

Table 2. Effect of fixed RELXILL parameters on the inner disk radius.

\begin{tabular}{lcc}
\hline Parameter value & $R_{\text {in }}\left(R_{\mathrm{ISCO}}\right)$ & $\Delta \chi^{2}$ \\
\hline$a=0$ & $36.6_{-16.3}^{*}$ & 0 \\
$q=2.5$ & $41.8_{-24.8}^{*}$ & 0.01 \\
$q=4$ & $46.7_{-23.6}^{*}$ & -0.01 \\
$E_{\text {cut }}=250$ & $49.0_{-16.8}^{*}$ & 3.4 \\
$E_{\text {cut }}=750$ & $49.5_{-29.1}^{*}$ & -2.5 \\
$A_{\mathrm{Fe}}=0.5$ & $48.0_{-27.8}^{*}$ & 22.1 \\
$A_{\mathrm{Fe}}=2.5$ & $38.4_{-17.7}^{*}$ & -16.8 \\
$R_{\text {out }}=1000 R_{\mathrm{ISCO}}$ & $37.0_{-17.2}^{*}$ & 0.1 \\
\hline
\end{tabular}

Notes. - The $\Delta \chi^{2}$ value is given with respect to our baseline model with $i=65^{\circ}$ (see Table 1 . An asterisk indicates that $R_{\text {in }}$ pegged at the model limit of $100 R_{\mathrm{ISCO}}$. Quoted errors reflect $1 \sigma$ confidence levels.

\subsubsection{Reflection fits with REFLIONX}

To ascertain that our inferred inner disk radius is not biased by our choice of model, we also fitted the reflection spectrum with REFLIONX (Ross \& Fabian 2005). This model was convolved with RELCONV to allow relativistic effects to shape the reflection spectrum. The REFLIONX model also assumes that the illuminating flux is supplied by power-law spectrum, which is a good approximation for IGR J17062-6143 (see Section 3.1). Setting $i=65^{\circ}, q=3$, and $A_{\mathrm{Fe}}=1$ yielded a good fit ( $\chi_{\nu}^{2}=0.99$ for 578 d.o.f.) with $R_{\text {in }}=53.2_{-20.0}^{*} R_{\text {ISCO }}$. This fit using REFLIONX is shown in Figure 1 (bottom). Modeling the reflection spectrum with REFLIONX also favors a truncated inner disk.

\subsection{Looking for a second power-law emission component}

The presence of a $\simeq 0.5 \mathrm{keV}$ black body in the spectrum of IGR J17062-6143 suggest that we likely observe radiation from the accretion-heated neutron star surface. As noted by Wijnands et al. (2015), this thermal emission may be associated with a hard emission tail that has an equal contribution to the flux in the 0.5-10 $\mathrm{keV}$ band as the thermal component. The hard tail should roll-over due to the spectral cutoff of the surface emission (D'Angelo et al. 2015). We might thus expect the presence of a hard power-law emission component in the spectrum of IGR J17062-6143, in addition to the observed thermal surface emission and the $\Gamma \simeq 2$ power law that is presumably emitted by the accretion flow.

Adding a second power law to our BBODYRAD+PEGPWRL model for the NuSTAR/Swift continuum gives a significant improvement $\left(\chi_{\nu}^{2}=1.09\right.$ for 579 d.o.f.; $F$-test probability $\left.\simeq 10^{-7}\right)$. The first power-law component has an index of $\Gamma_{1}=2.28 \pm 0.09$ and 
Table 3. Results from modeling the NuSTAR/Chandra reflection spectrum.

\begin{tabular}{lccc}
\hline Model parameter & RELXILL & RELXILL & REFLIONX \\
\hline & $A_{\mathrm{Fe}}=1$ & $A_{\mathrm{Fe}}$ free & $A_{\mathrm{Fe}}$ free \\
$C$ (MEG) & $1.09 \pm 0.01$ & $1.09 \pm 0.01$ & $1.09 \pm 0.01$ \\
$C$ (HEG) & $1.02 \pm 0.01$ & $1.02 \pm 0.01$ & $1.02 \pm 0.01$ \\
$N_{\mathrm{H}}\left(\times 10^{21} \mathrm{~cm}^{-2}\right)$ & $1.90 \pm 0.19$ & $1.68 \pm 0.11$ & $2.47 \pm 0.03$ \\
$k T_{\mathrm{bb}}(\mathrm{keV})$ & $0.50 \pm 0.01$ & $0.50 \pm 0.01$ & $0.48 \pm 0.01$ \\
$N_{\mathrm{bb}}(\mathrm{km} / 10 \mathrm{kpc})^{2}$ & $30.8 \pm 1.8$ & $35.4 \pm 1.2$ & $39.3 \pm 2.8$ \\
$\Gamma$ & $2.09 \pm 0.01$ & $2.04 \pm 0.01$ & $2.13 \pm 0.02$ \\
$R_{\text {in }}\left(R_{\mathrm{ISCO}}\right)$ & $20.4 \pm 4.4$ & $36.0 \pm 9.5$ & $36.0 \pm 9.5$ \\
$\log \xi$ & $3.04 \pm 0.05$ & $3.10 \pm 0.06$ & $2.56 \pm 0.10$ \\
$A_{\mathrm{Fe}}(\times$ Solar $)$ & $1.0 \mathrm{fix}$ & $5.0 \pm 0.5$ & $1.8 \pm 0.4$ \\
$R_{\mathrm{refl}}$ & $0.47 \pm 0.05$ & $0.26 \pm 0.03$ & - \\
$N_{\text {reff }}\left(\times 10^{-4}\right)$ & $2.52 \pm 0.10$ & $2.64 \pm 0.06$ & $0.002 \pm 0.001$ \\
$\chi_{\nu}^{2}($ dof $)$ & $1.47(4516)$ & $1.46(4515)$ & $1.46(4515)$ \\
\hline
\end{tabular}

Notes. - The constant factor $C$ was set to 1 for NuSTAR and left free for the Chandra data. Quoted errors are $1 \sigma$ confidence.

contributes $\simeq 77$ per cent to the unabsorbed flux in the $0.5-10 \mathrm{keV}$ band. The second power-law component is both fainter and harder: $\Gamma_{2}=1.11 \pm 0.37$ and $\mathrm{a} \simeq 2$ per cent contribution to the $0.5-10 \mathrm{keV}$ unabsorbed flux. The fractional contribution of the hard power law to the $0.5-10 \mathrm{keV}$ flux is considerably lower than that of the thermal emission ( $\simeq 21 \%$ ), contrary to that expected when both are due to accretion on to the neutron star surface. Moreover, the second, harder power-law component starts to dominate the spectrum only at energies $\gtrsim 50 \mathrm{keV}$. It is plausible, that the harder power-law component is trying to account for spectral residuals at higher energies (see Figure 1 top) that could also be due to un-modelled reflection (i.e. a Compton hump).

We also tried adding a second power-law component to a model that includes reflection (RELXILL with $i=65^{\circ}$ ). The powerlaw folded into RELXILL then has an index of $\Gamma_{1}=2.20 \pm 0.03$. The additional power-law component is harder with $\Gamma_{2}=1.25_{-0.50}^{+0.11}$ and improves the fit ( $\chi_{\nu}^{2}=0.99$ for 576 d.o.f.). However, an $F$-test suggests $\mathrm{a} \simeq 4 \times 10^{-4}$ probability $(\simeq 3.5 \sigma)$ that this improvement is due to chance. Moreover, this additional power-law component starts to dominate the X-ray spectrum only at $\gtrsim 65 \mathrm{keV}$, which is close to the upper end of the NUSTAR energy band where the SNR is low (Section 2.1). It is therefore not clear if an additional emission component, approximated by a power law, is indeed present in the broad-band spectrum of IGR J17062-6143. We note that the inclusion of a second power-law component in the reflection fits leaves the inner disk radius virtually unchanged $\left(R_{\mathrm{in}}=41.5_{-19.6}^{*} R_{\mathrm{ISCO}}\right)$.

\subsection{Chandra X-ray continuum and reflection spectrum}

Just as for the NuSTAR/Swift data, the Chandra spectrum is better described by a power law plus black body model $\left(\chi_{\nu}^{2}=1.53\right.$ for 3955 d.o.f.) than by a power law alone $\left(\chi_{\nu}^{2}=1.56\right.$ for 3957 d.o.f.; $F$-test probability $\left.\simeq 10^{-17}\right)$. We obtain $N_{\mathrm{H}}=(2.6 \pm 0.3) \times$ $10^{21} \mathrm{~cm}^{-2}, \Gamma=2.24 \pm 0.05, k T_{\mathrm{bb}}=0.48 \pm 0.01 \mathrm{keV}$, and $R_{\mathrm{bb}}=2.77 \pm 0.06(\mathrm{~km} / 5.0 \mathrm{kpc})^{2}$. This continuum fit is shown in Figure 3 (top). We measure a $0.5-10 \mathrm{keV}$ unabsorbed flux of $F_{0.5-10} \simeq 1.1 \times 10^{-10} \mathrm{erg} \mathrm{cm}^{-2} \mathrm{~s}^{-1}$. This corresponds to $L_{0.5-10} \simeq 3.2 \times 10^{35}(D / 5.0 \mathrm{kpc})^{2} \mathrm{erg} \mathrm{s}^{-1}$ and is a factor $\simeq 2$ higher than observed in 2015 with NuSTAR/Swift (Section 3.1.

Our continuum fit leaves residuals at energies of $\simeq 1-2$ and $\gtrsim 5 \mathrm{keV}$ (Figure 3, top). A zoom of the $0.5-2.5 \mathrm{keV}$ range is shown in Figure 4 The un-modeled structure above $5 \mathrm{keV}$ likely corresponds to a broadened $\mathrm{Fe}-\mathrm{K}$ line, as was seen in the $\mathrm{NuS}$ $T A R$ data and successfully modeled as disk reflection. The struc-
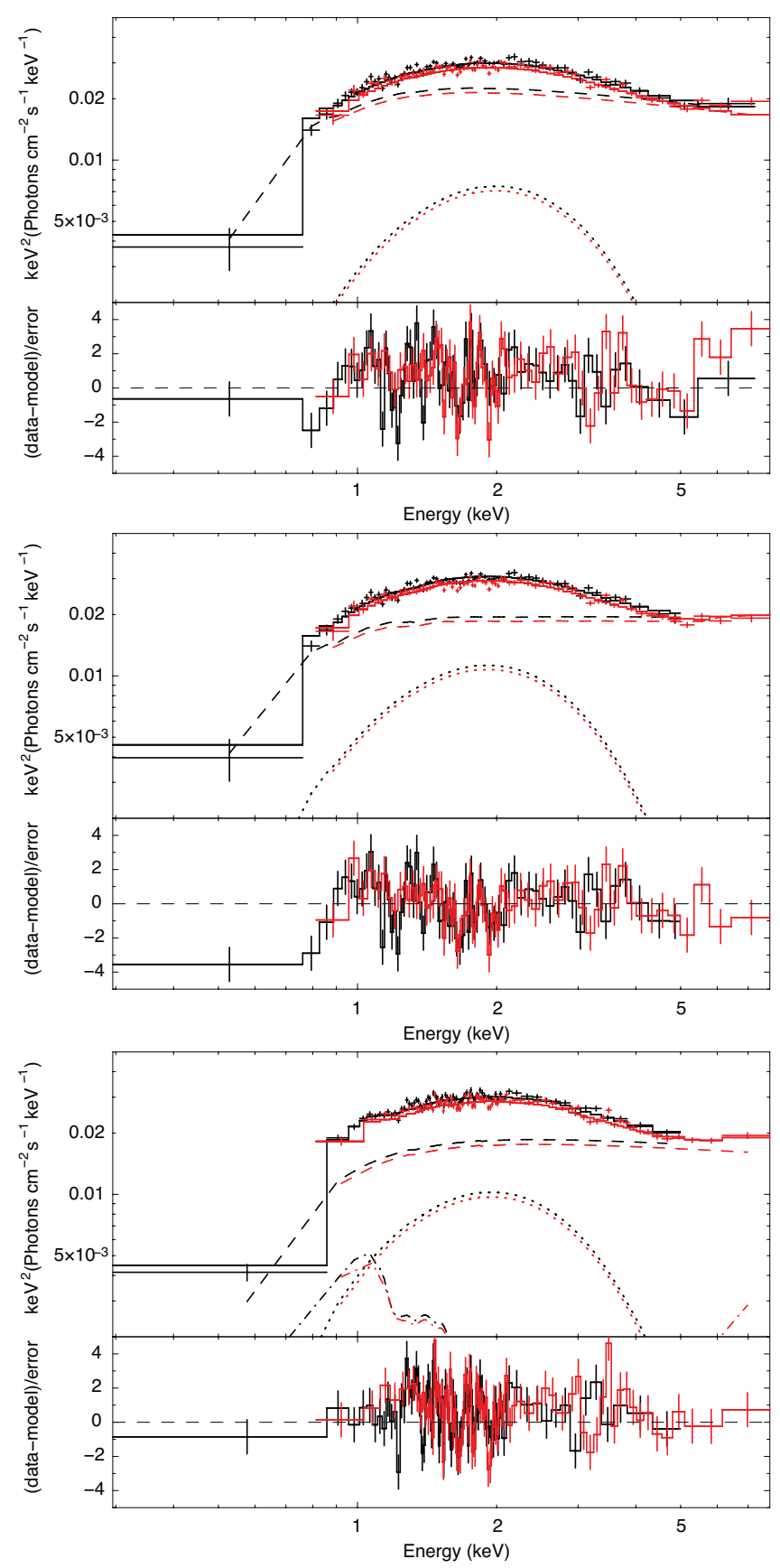

Figure 3. Fits to the Chandra MEG (black) and HEG (red) spectral data. Lower panels show the residuals in sigmas. Top: Continuum fit to an absorbed black body (dotted curve) and power law (dashed curve) model. Middle: Fit with the power-law component replaced by RELXILL to include relativistic reflection (dashed curve). Bottom: Fit with a black body (dotted curve) plus power law (dashed curve) continuum and REFLIONX (dasheddotted curve) as the relativistic reflection model.

ture near $1 \mathrm{keV}$ can potentially also arise from disk reflection (e.g. Fabian et al. 2009). To explore this possibility, we fitted the MEG and HEG data together with the NuSTAR spectrum.

We used RELXILL plus a black body with all parameters tied between the different instruments, but with constant factor included to allow for flux and calibration differences. Similarly to our $\mathrm{NuS}$ $T A R /$ Swift reflection fits, we fixed $q=3, R_{\text {out }}=500 R_{\mathrm{ISCO}}, a=0.3$, 


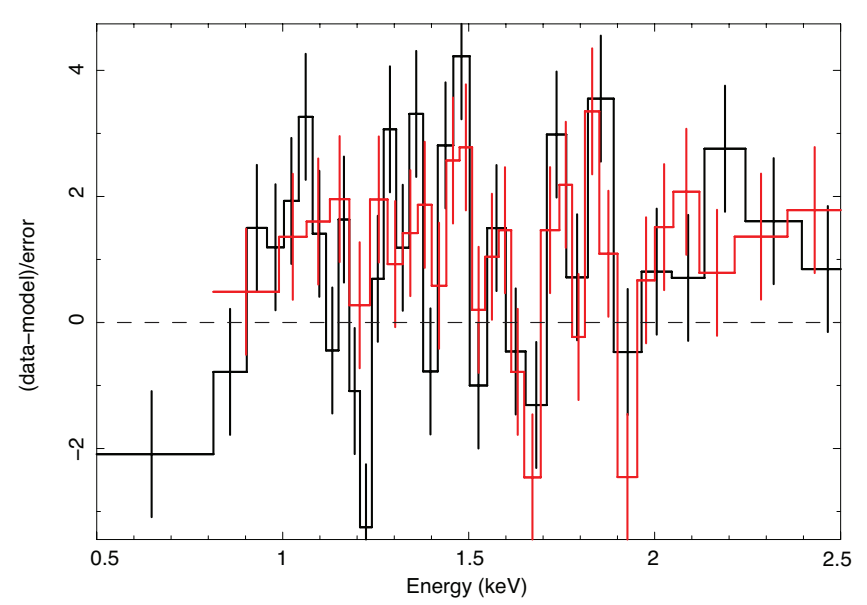

Figure 4. Zoom of the Chandra MEG (black) and HEG (red) data to model ratio for an absorbed power law plus black body continuum model.

$E_{\text {cut }}=500 \mathrm{keV}, i=65^{\circ}$ and $A_{\mathrm{Fe}}=1.0$ (see Section 3.2). The results of these fits are summarized in Table 3 We note that for these data the $\mathrm{Fe}$ abundance could be constrained when left free to vary. We obtained $A_{\mathrm{Fe}}=5.0 \pm 0.5$, which yielded an improved fit (with an $F$-test probability of $\simeq 10^{-7}$; see Table 3 .

As can be seen in Table 3, the results of fitting the Chandra and NUSTAR data together are similar to those obtained for the simultaneous NuSTAR/Swift data (cf. Table 1). The excess above $5 \mathrm{keV}$ in the Chandra data is accounted for by including relativistic reflection modeled as RELXILL, although residual structure remains near 1-2 keV (see Figure 3, bottom). We also tried fits with RELCONV*REFLIONX as our relativistic reflection model, where we again fixed $i=65^{\circ}, q=3, R_{\mathrm{out}}=500 R_{\mathrm{ISCO}}, a=0.3$, and $E_{\text {cut }}=500 \mathrm{keV}$, but left $A_{\mathrm{Fe}}$ free to vary. The results are included in Table 3. This reflection model seems to account better for the structure near $1 \mathrm{keV}$ in the Chandra data (Figure 3, bottom; see also Section 3.5). We note that the factor $\simeq 2$ flux difference between the Chandra and NUSTAR epochs could potentially result in differences in the reflection spectrum and could be a reason that some emission structure is not completely modeled.

\subsection{Chandra high-resolution X-ray spectrum}

\subsubsection{Line search}

We started our high-resolution spectral analysis with a phenomenological line search such as presented in Pinto et al. (2016). To this end we fitted the MEG and HEG data to an absorbed power law and black body continuum model, and added Gaussian lines with a fixed width of $1000 \mathrm{~km} \mathrm{~s}^{-1}$ over the 7-27 $\AA$ range with increments of $0.05 \AA$. The significance of the lines was computed by dividing the normalization by the $1 \sigma$ error. The results of our search are shown in Figure 5 as the black dotted line. This simple continuum description leaves several absorption (e.g. near 7.5 and $16 \AA$; $\simeq 1.65$ and $0.77 \mathrm{keV}$ ) and emission (e.g. near 12 and $13 \AA ; \simeq 1.03$ and $0.95 \mathrm{keV}$ ) features that appear to be significant at a $>3 \sigma$ level (see also the heavily binned spectral residuals in Figure 4).

Since the continuum modeling may affect the significance of any narrow spectral features, we repeated our line search after replacing the power-law component by RELXILL. For this reflection model we fixed all parameters except the normalization to the values obtained from fitting the Chandra and NuSTAR data together

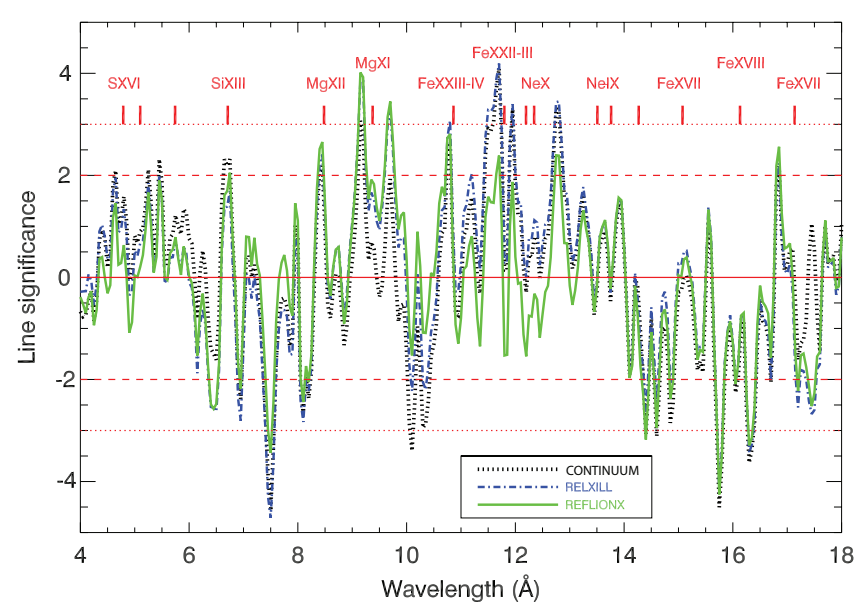

Figure 5. Significance of line features in the Chandra MEG and HEG data for different continuum models (see text for details). Positive and negative values indicate emission and absorption features, respectively. The rest wavelengths of abundant elements are indicated. The red dashed and dotted lines highlight $2 \sigma$ and $3 \sigma$ confidence levels, respectively.

with $A_{\mathrm{Fe}}$ free (Table 3). The result of this line search is shown as the blue dashed-dotted line in Figure 5 We mostly pick up the same emission and absorption features as for the continuum modeling, of which some (near $12 \AA$ ) appear more significant.

Finally, we ran our line-search routine after using RELCONV*RELFIONX, rather than RELXILL, as the reflection component. All parameters except the normalization were fixed to the values listed in Table 3 This reflection model seems to account for the narrow lines near near 12 and $13 \AA$ ( 11.03 and $0.95 \mathrm{keV})$, as is illustrated by the green curve in Figure 5 However, some of the other emission features remain also for this reflection model, e.g. near $9 \AA(\simeq 1.38 \mathrm{keV})$. Using RELFIONX also reduces the significance of the absorption line near $7.5 \AA(\simeq 1.65 \mathrm{keV})$. The only significant absorption features still present when using RELFIONX are lines near $16 \AA(\simeq 0.77 \mathrm{keV})$, which have similar significance for the three different spectral models that we tried. We note, however, that the signal-to-noise ratio is low at $\gtrsim 15 \AA(\lesssim 0.8 \mathrm{keV})$ and the continuum is less well constrained.

The significances calculated in this analysis implicitly assume that the lines are at rest. If the lines are blue/redshifted this adds to the number of trials and hence decreases the significance. This may particularly affect the significance of the absorption lines, which appear to require a significant blueshift to correspond to any abundant element (see Section 3.5.2). Most of the emission lines, on the other hand, could be consistent with near-rest frame wavelengths of abundant elements, hence the significances calculated here should be a good approximation. Running our line-detection search for line widths of $250 \mathrm{~km} \mathrm{~s}^{-1}$ and $5000 \mathrm{~km} \mathrm{~s}^{-1}$, yielded a similar picture as that presented above for $1000 \mathrm{~km} \mathrm{~s}^{-1}$.

Comparing the location of the various emission/absorption lines to the effective area of the MEG and HEG shows that none of the prominent features correspond to instrumental edges. Furthermore, we ran our line-search algorithm on Chandra gratings data of the blazars H 1821+643 (obsIDs 1599, 2186, 2310), PKS 2155-304 (ObsIDs 337, 3167, 9712), and Mrk 421 (obsIDs 10663, 4148,4149 ), which provided similar statistics as our data set and are not expected to show narrow spectral features. Indeed, we did not find any lines of comparable magnitude. Therefore, there are no 


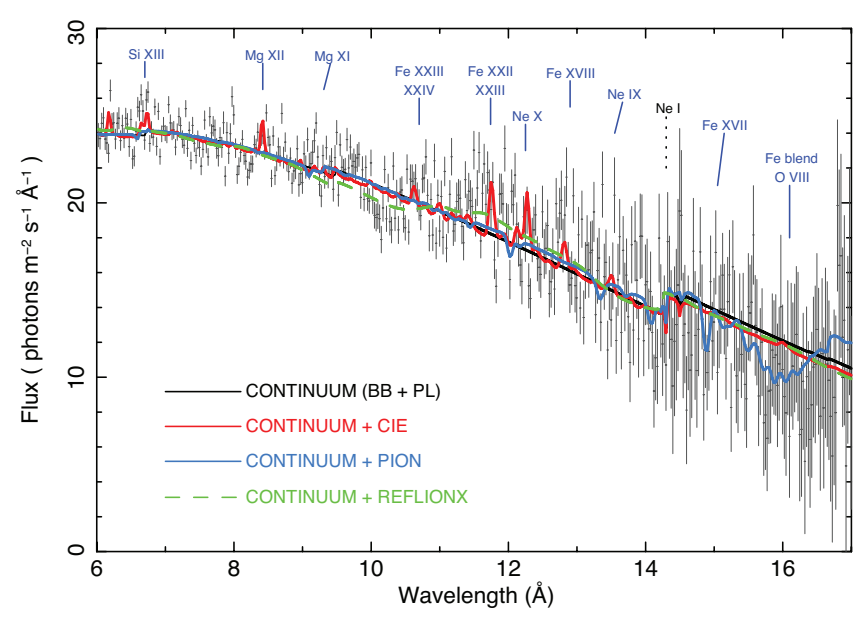

Figure 6. Zoom of the Chandra/MEG data fitted to different spectral models (see text for details). The position of several rest-frame transitions commonly seen in LMXBs (as well as active galactic nuclei) are indicated.

obvious indications that the narrow features picked up by our line search are instrumental or due to statistical fluctuations.

\subsubsection{Spectral modeling}

Assuming that the discrete emission and absorption lines found in our simple line search are real, we explored spectral models to probe what physical processes could cause these features. Unfortunately, due to the low flux and low significance of the lines, we cannot present a definitive solution. We explore different possibilities below and discuss some physical implications in Section 4 Since the fits are intended to be exploratory and are non-unique, we do not give errors on the fit parameters. In Figure 6 we show a zoom of the MEG data that captures our results.

The black curve in Figure 6 shows a fit to a simple continuum consisting of a black body and a power law, which yields a $C$-stat value of 1447 for 1211 dof. As shown in Section 3.5.1 such a model would suggest the presence of some absorption and emission features. To explore the origin of the emission features, we added a CIE component in our SPEX fits, which describes the spectrum of a plasma in collisional-ionisation equilibrium. We adopted Solar abundances from Lodders \& Palme (2009). The CIE model has three free parameters: the plasma temperature $k T_{\text {cie }}$, the line broadening $\sigma_{\text {cie }}$, and a normalization. We obtain $k T_{\text {cie }} \simeq 1 \mathrm{keV}$, $\sigma_{\text {cie }} \simeq 500-1000 \mathrm{~km} \mathrm{~s}^{-1}$, and $C$-stat $=1402$ for 1208 d.o.f. This fit is shown as the red curve in Figure 6 A collisional-ionized plasma would be able to account for several narrow emission lines.

To model the absorption features, we tried adding a photoionized absorber, PION, to an absorbed power law plus black body continuum. The PION model has four fit parameters: the ionization parameter $\log \xi$, the line broadening $\sigma_{\text {pion }}$, the line-of-sight velocity $v_{\text {pion }}$, and a normalization. We again adopted Solar abundances (Lodders \& Palme 2009). We obtain $\log \xi \simeq 2, \sigma_{\text {pion }} \simeq 500$ $1000 \mathrm{~km} \mathrm{~s}^{-1}, v_{\text {pion }} \simeq-(2000-3500) \mathrm{km} \mathrm{s}^{-1}(\simeq 0.01 c)$, and $C$ stat $=1422$ for 1207 d.o.f. The best candidates for the $15.75 \AA$ and weaker $16.25 \AA$ absorption features are "hot" OvIII or "cooler" FeXVI (or lower Fe ions). The line broadening is similar to that obtained for the CIE plasma model, which suggests that the emission and absorption lines could possibly arise from the same physical location. This fit is shown as the blue line in Figure 6 Including photo-ionized absorption reproduces a relatively broad absorption feature near $16 \AA(\simeq 0.77 \mathrm{keV})$ that was also found in our line search (Figure 5, see also Figure 4). The obtained line-of-sight velocity is negative, which would point to outflowing gas.

The green dashed curve in Figure 6 shows a fit that includes reflection modeled as REFLIONX, which yields $C$-stat $=1417$ for 1207 dof. As this raises the flux near $12 \AA(\simeq 1 \mathrm{keV})$ it can account for part of the emission excess. Nevertheless, some narrower emission features may not be accounted for (Section 3.5.1). If real, this un-modelled structure could perhaps be due to reflection that requires a narrower core (i.e. less relativistic blurring; more distant reflection) than supplied by the input model, or a different emission mechanism. Adding a CIE model component results in $\Delta C$-stat $=-$ 27 , and may indeed indicate that there are emission features that are not fully modeled by a single reflection zone.

\section{DISCUSSION}

In this work we presented NuSTAR, Swift and Chandra observations of the neutron star LMXB IGR J17062-6143, which has been persistently accreting at $L_{\mathrm{X}} \simeq 10^{-3} L_{\mathrm{Edd}}$ since 2006 . The aim of this study was to gain more insight into the morphology of lowlevel accretion flows, and the nature of neutron star LMXBs that are able to accrete at these low rates for several years.

The 0.5-79 keV NuSTAR/Swift spectrum of IGR J17062-6143 can be modeled as a combination of a $\Gamma \simeq 2$ power-law component and a soft, thermal component that can be described by a $k T \simeq 0.5 \mathrm{keV}$ black body. This continuum spectral shape is very similar to that inferred from XMM-Newton data obtained for a few neutron star LMXBs at $L_{\mathrm{X}} \simeq 10^{-4} L_{\mathrm{Edd}}$ (e.g. Armas Padilla et al. 2011, 2013a.b; Degenaar et al. 2013b). The temperature of the black body component appears to be too high for the measured Xray luminosity, and the inferred radius to small, to be from the accretion disk. The thermal emission is therefore more likely coming from (part of) the neutron star surface, which is expected to become visible when moving to low $L_{\mathrm{X}}$. We do not seem to detect the hard emission tail that is thought to be associated with surface accretion (e.g. Deufel et al. 2001; D'Angelo et al. 2015; Wijnands et al. 2015). Possibly, at $L_{\mathrm{X}} \simeq 10^{-3} L_{\mathrm{Edd}}$ this component is difficult to disentangle from the emission of the accretion flow itself.

The spectral data of IGR J17062-6143 show a broadened $\mathrm{Fe}-\mathrm{K}$ line at $\simeq 6-7 \mathrm{keV}$. Such a feature has never been detected for a neutron star LMXB accreting at $L_{\mathrm{X}} \lesssim 10^{-2} L_{\mathrm{Edd}}$ before (despite the availability of high-quality data; e.g. Armas Padilla et al. 2013a; Lotti et al. 2016), but it is commonly seen in brighter LMXBs. Assuming that the line is due to relativistic disk reflection, we modeled the spectral data with RELXILL. This suggests that the inner disk radius was truncated away from the ISCO, at $R_{\text {in }} \gtrsim 20 R_{\text {ISCO }}\left(\gtrsim 100 \mathrm{GM} / \mathrm{c}^{2}\right.$ or $\gtrsim 225 \mathrm{~km} ; 1 \sigma$ confidence). Due to the low flux of IGR J17062-6143, however, the data quality is not good enough to rule out a location at the ISCO at $>3 \sigma$ significance. Nevertheless, it would not be surprising to find a truncated disk at $L_{\mathrm{X}} \lesssim 10^{-2} L_{\mathrm{Edd}}$ and it is therefore interesting to further explore this (Section 4.1).

Our high-resolution Chandra gratings data of IGR J170626143 reveal hints of discrete emission and absorption lines in the $\simeq 0.5-1.5 \mathrm{keV}$ energy range. This includes what appears to be a collection of narrow emission lines near $12 \AA(\simeq 1 \mathrm{keV})$ that can be modelled as disk reflection or a collisionally-ionized plasma. Unfortunately the significance of the narrow spectral features is low $(\lesssim 4.5 \sigma)$ and depends on the underlying continuum/reflection spec- 
trum. However, since this is the first gratings data of a neutron star LMXB accreting as low as $L_{\mathrm{X}} \simeq 10^{-3} L_{\mathrm{Edd}}$, it is interesting to explore plausible physical scenarios that could account for these lines (Section 4.3 and 4.5). The strongest and most robust feature found in our simple line search is a relatively broad feature near $16 \AA$ $(\simeq 0.77 \mathrm{keV})$, which has a significance of $\simeq 4 \sigma$ for all spectral models that we explored (not accounting for trials). If the absorption is real, it can can be modelled as an outflowing photo-ionized plasma with a line-of-sight velocity of $\simeq 2000-3500 \mathrm{~km} \mathrm{~s}^{-1}(\simeq 0.01 c)$. An outflow might be expected if the inner disk in IGR J17062-6143 is truncated due to the formation of a radiatively-inefficient accretion flow (e.g. Narayan \& Yi 1994; Blandford \& Begelman 1999; Narayan 2005), or if the magnetosphere of the neutron star is acting as a propeller (e.g. Illarionov \& Sunyaev 1975); Romanova et al. 2009; Papitto \& Torres 2015).

\subsection{Disk truncation at the magnetospheric boundary?}

If the inner disk is indeed truncated at $R_{\text {in }} \gtrsim 100 \mathrm{GM} / \mathrm{c}^{2}$ in IGR $\mathrm{J} 17062-6143$, this is a factor $\gtrsim 7$ higher than typically found for non-pulsating neutron star LMXBs accreting at $L_{\mathrm{X}} \gtrsim 10^{-2} L_{\mathrm{Edd}}$ ( $R_{\text {in }} \simeq 5-15 G M / c^{2}$; e.g. Cackett et al. 2010b; Egron et al. 2013; Miller et al. 2013; Degenaar et al. 2015; Di Salvo et al. 2015; Ludlam et al. 2016; (Sleator et al.2016). It is also a factor $\gtrsim 3$ higher than inferred for several (millisecond) $\mathrm{X}$-ray pulsars that accrete at $L_{\mathrm{X}} \gtrsim 10^{-2} L_{\mathrm{Edd}}\left(R_{\mathrm{in}} \simeq 15-30 \mathrm{GM} / \mathrm{c}^{2}\right.$; e.g. Miller et al. 2011; Papitto et al. 2013; King et al. 2016; Pintore et al. 2016).

The black hole LMXB GX 339-4 shows a much narrower Fe$\mathrm{K}$ line at $\simeq 10^{-3} L_{\mathrm{Edd}}$ than at higher accretion luminosity. This can be interpreted as the inner disk receding from the ISCO to $R_{\text {in }} \simeq 35 \mathrm{GM} / \mathrm{c}^{2}$ as the mass-accretion rate drops, presumably due to disk evaporation (Tomsick et al. 2009). Our study suggests a larger truncation radius for IGR J17062-6143 at similar Eddingtonscaled accretion rate. Disk evaporation should operate in neutron star LMXBs too, although it is expected to set in at lower $L_{\mathrm{X}}$ than for black holes because soft photons emitted from the stellar surface cool the hot flow (e.g. Narayan \& Yi 1995). If the inner disk in IGR J17062-6143 is indeed further out than in GX 339-4, this might point to a different truncation mechanism.

We note that there is degeneracy in inferring a truncated disk from reflection modeling (e.g. Fabian et al. 2014). In particular, in case of GX 339-4 it has been pointed out that the narrowing of the Fe-K line with decreasing $L_{\mathrm{X}}$ could also be due to the illuminating $\mathrm{X}$-ray source moving away, i.e. an increasing height of the corona in a "lamp-post" geometry (Dauser et al. 2013). It is, however, not obvious that in neutron star LMXBs the accretion disk is also illuminated by a corona (rather than e.g. the boundary layer) and hence that a lamp-post geometry would apply for IGR J17062-6143.

In neutron star LMXBs, it is also possible that the stellar magnetic field truncates the inner accretion disk. In fact, a magneticallyinhibited accretion flow has been proposed as a possible explanation for the sustained low accretion rate of some VFXBs like IGR J17062-6143 (Heinke et al. 2009, 2015; Degenaar et al. 2014b). If the blue-shifted absorption in the Chandra data is real, this could possibly form a consistent physical picture in which the accretion flow is stopped at the magnetospheric boundary that acts as a propeller. This is an interesting scenario because the inferred inner disk radius would then provide constraints on the magnetic field strength and spin period of the neutron star.

\subsection{Estimates of the neutron star magnetic field strength}

If the inner accretion disk in IGR J17062-6143 is truncated at the magnetospheric radius, we can estimate the magnetic field strength. To this end we use equation (1) from Cackett et al. (2009), which is based on the derivations of Ibragimov \& Poutanen (2009), to write the following expression for the magnetic field strength:

$$
\begin{aligned}
B= & 1.2 \times 10^{5} k_{\mathrm{A}}^{-7 / 4}\left(\frac{R_{\mathrm{in}}}{G M / c^{2}}\right)^{7 / 4}\left(\frac{M}{1.4 \mathrm{M}_{\odot}}\right)^{2} \frac{D}{5 \mathrm{kpc}} \\
& \times\left(\frac{R}{10^{6} \mathrm{~cm}}\right)^{-3}\left(\frac{f_{\text {ang }}}{\eta} \frac{F_{\text {bol }}}{10^{-9} \mathrm{erg} \mathrm{cm}^{-2} \mathrm{~s}^{-1}}\right)^{1 / 2} \mathrm{G},
\end{aligned}
$$

where $f_{\text {ang }}$ is an anisotropy correction factor (which is close to unity; Ibragimov \& Poutanen 2009), $k_{\mathrm{A}}$ a geometry coefficient (expected to be $\simeq 0.5-1.1$; Psaltis \& Chakrabarty 1999; Long et al. 2005; Kluźniak \& Rappaport 2007), and $\eta$ the accretion efficiency. We use $D=5 \quad \mathrm{kpc}, \quad M=1.4 \quad \mathrm{M}_{\odot}, \quad R=10 \quad \mathrm{~km}$, $R_{\text {in }} \gtrsim 100 \mathrm{GM} / \mathrm{c}^{2}$, and conservatively assume that the bolometric flux is equal to the $0.5-79 \mathrm{keV}$ flux determined from our joint $N u S$ TAR/Swift fits (i.e. $F_{\mathrm{bol}}=F_{0.5-79} \simeq 1.2 \times 10^{-10} \mathrm{erg} \mathrm{cm}^{-2} \mathrm{~s}^{-1}$ ). Furthermore, we assume $f_{\text {ang }}=1, k_{\mathrm{A}}=1$, and $\eta=0.1$. We then obtain $B \gtrsim 4 \times 10^{8} \mathrm{G}$ for IGR J17062-6143. This is a factor of a few higher than typical estimates for neutron stars in LMXBs, although within the maximum allowable range determined in a recent analysis of the coherent timing properties of several millisecond X-ray pulsars (Mukheriee et al. 2015).

\subsection{A propeller-driven outflow?}

If the accretion disk in IGR J17062-6143 is indeed truncated and this is due to the magnetic field of the neutron star, the rotating magnetosphere may act as a propeller (e.g. Illarionov \& Sunvaev 1975; Lovelace et al. 1999; Romanova et al. 2009; Papitto \& Torres 2015). Magnetohydrodynamic simulations show that an active propellor can cause a two-component outflow consisting of an axial jet and a conical wind (e.g. Romanova et al. 2009). The wind component has a high density, outflow velocity of $\simeq 0.03 c-0.1 c$, and is shaped like a thin conical shell with a halfopening angle of $\simeq 30^{\circ}-40^{\circ}$. The jet component has a lower density and a higher outflow velocity $(\simeq 0.4 c-0.6 c)$.

If the blue-shifted absorption in our Chandra data is real, a line-of-sight velocity of $\simeq 0.01 c$ could potentially be consistent with a wind driven by an active propeller. Interestingly, a small subgroup of neutron star LMXBs that appear to exhibit propeller stages, the transitional millisecond radio pulsars (e.g. Archibald et al. 2009; Papitto et al. 2014; Papitto \& Torres 2015), seem to have more luminous radio jets than other neutron star LMXBs (Deller et al. 2015). If a propeller is operating in IGR J17062-6143, it may thus be expected to exhibit a strong radio jet too.

We note that even if the magnetic field is truncating the inner accretion disk, it is not necessary that a propeller is operating. Another possibility is a "trapped disk" morphology (e.g. D’Angelo \& Spruit 2010, 2012). In the propellor scenario, strong outflows are formed and little matter accretes on to the neutron star so that the accretion flow may dominate the overall X-ray luminosity. For a trapped disk, however, only a weak outflow is expected and considerable amounts of gas can still accrete on to the neutron star magnetic poles, which may dominate the overall X-ray luminosity. 


\subsection{Estimates of the neutron star spin period}

If the blue-shifted absorption in our Chandra data is real and due to a propeller-driven outflow, the assumption that the inner accretion disk is truncated at the magnetospheric boundary allows to put some constraints on the neutron star spin period. A neutron star is thought to be in the propellor regime when the magnetospheric radius is larger than the co-rotation radius. At this radius the $\mathrm{Ke}$ plerian orbital velocity of the matter equals the rotational velocity of the neutron star, i.e. $R_{\mathrm{co}}=\left(G M P_{\mathrm{s}}^{2} / 4 \pi^{2}\right)^{1 / 3}$, where $P_{\mathrm{s}}$ is the spin period of the neutron star. Assuming that the inner disk radius is truncated by the magnetosphere, i.e. $R_{\mathrm{m}}=R_{\mathrm{in}} \gtrsim 100 \mathrm{GM} / \mathrm{c}^{2}$ ( $\simeq 225 \mathrm{~km}$ ), the requirement that $R_{\mathrm{m}}>R_{\text {co }}$ suggests that IGR $\mathrm{J} 17062-6143$ is in the propellor regime if $P_{\mathrm{s}} \lesssim 19 \mathrm{~ms}$.

We seem to detect thermal emission from the stellar surface in our X-ray spectra and two thermonuclear X-ray bursts have been detected from IGR J17062-6143 (Degenaar et al.2013a; Iwakiri et al. 2015; Negoro et al. 2015). This implies that at least some matter must be able to accrete on to the neutron star. The accretion disk must therefore lie within the light cylinder radius, where the rotational velocity of the magnetic field lines reaches the speed of light, i.e. $R_{\mathrm{lc}}=c / \Omega$ with $\Omega=2 \pi / P_{\mathrm{s}}$ being the angular velocity. If $R_{\text {in }} \gtrsim 100 \mathrm{GM} / \mathrm{c}^{2}(\simeq 225 \mathrm{~km})$, this would suggest that $P_{\mathrm{s}} \gtrsim 4.7 \mathrm{~ms}$.

If the accretion disk is truncated by the magnetic field and a propellor operates, that would require $P_{\mathrm{s}} \simeq 4.7-19 \mathrm{~ms}$ for IGR J17062-6143. This is within the typical range of spin periods measured for neutron stars LMXBs from coherent X-ray pulsations or burst oscillations (1.6-10 ms; e.g. Patruno 2010b, for a list).

\subsection{Low-energy narrow X-ray emission lines}

Binning the Chandra/HETG data reveals an emission feature near $1 \mathrm{keV}$. A broad line around the same energy was detected in the Swift/XRT data obtained during the energetic X-ray burst of IGR J17062-6143 in $2012\left(E_{1}=1.018 \pm 0.004 \mathrm{keV}\right.$, and EW= $105 \pm 3 \mathrm{eV}$; Degenaar et al. 2013a). It was interpreted as Fe-L or $\mathrm{Ne} \mathrm{X}$ emission arising from irradiation of relatively cold gas orbiting at a distance of $\simeq 10^{3} \mathrm{~km}\left(\simeq 500 \mathrm{GM} / \mathrm{c}^{2}\right)$ from the neutron star (by assuming that the line was rotationally-broadened by gas moving in Keplerian orbits). Broad emission lines near $1 \mathrm{keV}$ have been detected in the accretion spectra of a number of other neutron star LMXBs (e.g. Vrtilek et al. 1991; Kuulkers et al. 1997; Díaz Trigo et al. 2006; Cackett et al. 2010b; Papitto et al. 2013).

Exploiting the high spectral resolution of the HETG, we found that in IGR J17062-6143 the emission feature near $1 \mathrm{keV}(\simeq 12 \AA)$ may be resolved into a number of narrow lines. Narrow emission lines also appear to be present at other energies (e.g. near 9 and $10 \AA ; \simeq 1.38$ and $1.24 \mathrm{keV}$ ). High-resolution observations of some other neutron star LMXBs revealed complexes of narrow emission lines at low energies, typically consistent with being at rest (e.g. Cottam et al. 2001a, b; Schulz et al. 2001; Beri et al. 2015). Proposed explanations include a pulsar-driven disk wind or photoionized emission from a thickened structure in the accretion disk (e.g. the impact point where the gas stream from the companion hits the outer accretion disk).

In case of IGR J17062-6143, the strongest narrow emission line is located at $\simeq 11.6 \AA$ ( $\simeq 1.07 \mathrm{keV})$. If real, it could correspond to $\mathrm{Fe}-\mathrm{L}$ at rest. This would render a collisionally-ionized plasma more likely; photo-ionized gas lines from lower-Z elements (e.g. $\mathrm{O}, \mathrm{Ne}$ ) should be stronger than Fe-L, which doesn't seem to be the case for our data. Perhaps shocks resulting from the accretion flow running into the magnetosphere or from matter impacting the magnetic poles could give rise to collisionally-ionized emission in this neutron star LMXB. Alternatively, this line could correspond to Ne $\mathrm{X}$ blue-shifted by $\simeq 0.045 c\left(\simeq 13.5 \times 10^{3} \mathrm{~km} \mathrm{~s}^{-1}\right)$, which would be indicative of an outflow. A third, perhaps more likely, possibility is that the emission lines are due to reflection. However, a single reflection component that also fits the $\mathrm{Fe}-\mathrm{K}$ line seems to leave excess emission near $1 \mathrm{keV}$. This could indicate that there are multiple reflection zones, or that different emission mechanisms are responsible for the different lines.

\section{ACKNOWLEDGEMENTS}

ND is supported by an NWO Vidi grant and an EU Marie Curie Intra-European fellowship (contract no. FP-PEOPLE-2013-IEF627148). ND acknowledges valuable discussions with Anne Lohfink, Victor Doroshenko, and Caroline D'Angelo. CP and ACF are supported by ERC Advanced Grant Feedback 340442. JMM acknowledges support from the Chandra guest observer program. DA acknowledges support from the Royal Society. RW is supported by an NWO Top grant, module 1 . We thank the referee for useful comments. This work is based on data from the NUSTAR mission, a project led by California Institute of Technology, managed by the Jet Propulsion Laboratory, and funded by NASA. We thank Neil Gehrels and the Swift duty scientists for rapid scheduling of observations and acknowledge the use of the Swift public data archive.

\section{REFERENCES}

Allen J. L., Linares M., Homan J., Chakrabarty D., 2015, ApJ, 801, 10 Alpar M., Cheng A., Ruderman M., Shaham J., 1982, Nature, 300, 728 Archibald A. et al., 2009, Science, 324, 1411

Armas Padilla M., Degenaar N., Wijnands R., 2013a, MNRAS, 434, 1586 Armas Padilla M., Wijnands R., Degenaar N., 2013b, MNRAS, 436, L89 Armas Padilla M., Wijnands R., Altamirano D., Méndez M., Miller J., Degenaar N., 2014, MNRAS, 439, 3908

Armas Padilla M., Degenaar N., Patruno A., Russell D., Linares M., Maccarone T., Homan J., Wijnands R., 2011, MNRAS, 417, 659

Arnason R. M., Sivakoff G. R., Heinke C. O., Cohn H. N., Lugger P. M., 2015, ApJ, 807, 52

Arnaud K., 1996, in G. Jacoby, J. Barnes, eds, Astronomical Data Analysis Software and Systems V. Astronomical Society of the Pacific Conference Series, Vol. 101, p. 17

Bahramian A. et al., 2014, ApJ, 780, 127

Bassa C. et al., 2008, ATel, 1575

Beri A., Paul B., Dewangan G. C., 2015, MNRAS, 451, 508

Bernardini F., Cackett E., Brown E., D’Angelo C., Degenaar N., Miller J., Reynolds M., Wijnands R., 2013, MNRAS, 436, 2465

Bhattacharya D., van den Heuvel E., 1991, Phys. Rev., 203, 1

Blandford R., Begelman M., 1999, MNRAS, 303, L1

Braje T. M., Romani R. W., Rauch K. P., 2000, ApJ, 531, 447

Cackett E., Altamirano D., Patruno A., Miller J., Reynolds M., Linares M., Wijnands R., 2009, ApJL, 694, L21

Cackett E., Brown E., Miller J., Wijnands R., 2010a, ApJ, 720, 1325

Cackett E., Brown E., Degenaar N., Miller J., Reynolds M., Wijnands R., 2013, MNRAS, 433, 1362

Cackett E. et al., 2010b, ApJ, 720, 205

Campana S., 2009, ApJ, 699, 1144

Campana S., Mereghetti S., Stella L., Colpi M., 1997, A\&A, 324, 941

Chakrabarty D. et al., 2014, ApJ, 797, 92

Chelovekov I., Grebenev S., 2007, Astronomy Letters, 33, 807

Chiang C. Y. et al., 2016, ApJ, 821, 105

Churazov E. et al., 2007, A\&A, 467, 529 
Cottam J., Kahn S. M., Brinkman A. C., den Herder J. W., Erd C., 2001a, A\&A, 365, L277

Cottam J., Sako M., Kahn S. M., Paerels F., Liedahl D. A., 2001b, ApJL, 557, L101

D’Angelo C. R., Spruit H. C., 2010, MNRAS, 406, 1208

D'Angelo C. R., Spruit H. C., 2012, MNRAS, 420, 416

D’Angelo C. R., Fridriksson J. K., Messenger C., Patruno A., 2015, MN-

RAS, 449, 2803

Dauser T., Wilms J., Reynolds C. S., Brenneman L. W., 2010, MNRAS, 409, 1534

Dauser T., Garcia J., Wilms J., Böck M., Brenneman L. W., Falanga M., Fukumura K., Reynolds C. S., 2013, MNRAS, 430, 1694

Degenaar N., Wijnands R., 2009, A\&A, 495, 547

Degenaar N., Altamirano D., Wijnands R., 2012a, ATel, 4219

Degenaar N., Miller J., Wijnands R., Altamirano D., Fabian A., 2013a, ApJL, 767, L37

Degenaar N., Wijnands R., Miller J., 2013b, ApJL, 767, L31

Degenaar N., Miller J. M., Harrison F. A., Kennea J. A., Kouveliotou C., Younes G., 2014a, ApJL, 796, L9

Degenaar N., Miller J. M., Chakrabarty D., Harrison F. A., Kara E., Fabian A. C., 2015, MNRAS, 451, L85

Degenaar N. et al., 2010, MNRAS, 404, 1591

Degenaar N. et al., 2012b, A\&A, 540, A22

Degenaar N. et al., 2014b, ApJ, 792, 109

Del Santo M., Sidoli L., Mereghetti S., Bazzano A., Tarana A., Ubertini P., 2007, A\&A, 468, L17

Deller A. T. et al., 2015, ApJ, 809, 13

Deufel B., Dullemond C., Spruit H., 2001, A\&A, 377, 955

Di Salvo T. et al., 2015, MNRAS, 449, 2794

Díaz Trigo M., Parmar A. N., Boirin L., Méndez M., Kaastra J. S., 2006, A\&A, 445, 179

Dubus G., Lasota J. P., Hameury J. M., Charles P., 1999, MNRAS, 303, 139

Dubus G., Hameury J. M., Lasota J. P., 2001, A\&A, 373, 251

Egron E. et al., 2013, A\&A, 550, A5

Fabian A., Rees M., Stella L., White N., 1989, MNRAS, 238, 729

Fabian A. C., Ross R. R., 2010, SSRv, 157, 167

Fabian A. C., Parker M. L., Wilkins D. R., Miller J. M., Kara E., Reynolds

C. S., Dauser T., 2014, MNRAS, 439, 2307

Fabian A. C. et al., 2009, Nature, 459, 540

Finger M., Koh D., Nelson R., Prince T., Vaughan B., Wilson R., 1996, Nature, 381, 291

Frank J., King A., Raine D., 2002, Accretion Power in Astrophysics: Third Edition

García J., Dauser T., Reynolds C. S., Kallman T. R., McClintock J. E.,

Wilms J., Eikmann W., 2013, ApJ, 768, 146

García J. et al., 2014, ApJ, 782, 76

George I. M., Fabian A. C., 1991, MNRAS, 249, 352

Hameury J. M., Lasota J. P., 2016, arXiv:1607.06394

Hands A., Warwick R., Watson M., Helfand D., 2004, MNRAS, 351, 31

Heinke C., Cohn H., Lugger P., 2009, ApJ, 692, 584

Heinke C. et al., 2010, ApJ, 714, 894

Heinke C. O., Bahramian A., Degenaar N., Wijnands R., 2015, MNRAS, 447, 3034

Ibragimov A., Poutanen J., 2009, MNRAS, 400, 492

Illarionov A., Sunyaev R., 1975, A\&A, 39, 185

in 't Zand J., Jonker P., Markwardt C., 2007, A\&A, 465, 953

in 't Zand J., Jonker P., Bassa C., Markwardt C., Levine A., 2009, A\&A, 506,857

Iwakiri W., Keek L., Serino M., Strohmayer T., in't Zand J., Kuulkers E., Sakamoto T., 2015, ATel, 8253

Jonker P., Keek L., 2008, ATel, 1643

King A., Wijnands R., 2006, MNRAS, 366, L31

King A. L. et al., 2016, ApJL, 819, L29

Kluźniak W., Rappaport S., 2007, ApJ, 671, 1990

Kuulkers E., Parmar A. N., Owens A., Oosterbroek T., Lammers U., 1997, A\&A, 323, L29
Kuulkers E., den Hartog P., in 't Zand J., Verbunt F., Harris W., Cocchi M., 2003, A\&A, 399, 663

Kuulkers E., in 't Zand J., Lasota J. P., 2009, A\&A, 503, 889

Lasota J. P., 2001, NewAR, 45, 449

Lodders K., Palme H., 2009, Meteoritics and Planetary Science Supplement, 72, 5154

Long M., Romanova M. M., Lovelace R. V. E., 2005, ApJ, 634, 1214

Lotti M., et al., 2016, ApJ, 822, 57

Lovelace R. V. E., Romanova M. M., Bisnovatyi-Kogan G. S., 1999, ApJ, 514,368

Ludlam R. M. et al., 2016, ApJ, 824, 37

Matt G., Perola G. C., Piro L., 1991, A\&A, 247, 25

Menou K., Hameury J. M., Lasota J. P., Narayan R., 2000, MNRAS, 314, 498

Miller J., Maitra D., Cackett E., Bhattacharyya S., Strohmayer T., 2011, ApJL, 731, L7

Miller J. et al., 2013, ApJL, 779, L2

Miller M. C., Lamb F. K., Cook G. B., 1998, ApJ, 509, 793

Mukherjee D., Bult P., van der Klis M., Bhattacharya D., 2015, MNRAS, 452, 3994

Muno M., Pfahl E., Baganoff F., Brandt W., Ghez A., Lu J., Morris M., 2005, ApJL, 622, L113

Narayan R., 2005, A\&AS, 300, 177

Narayan R., Yi I., 1994, ApJL, 428, L13

Narayan R., Yi I., 1995, ApJ, 452, 710

Negoro H. et al., 2015, ATel, 8241

Ng C., Díaz Trigo M., Cadolle Bel M., Migliari S., 2010, A\&A, 522, A96

Papitto A., Torres D. F., 2015, ApJ, 807, 33

Papitto A., Torres D., Li J., 2014, MNRAS, 438, 2105

Papitto A. et al., 2013, MNRAS, 429, 3411

Patruno A., 2010a, in "Proceedings of High Time Resolution Astrophysics - The Era of Extremely Large Telescopes”, arXiv:1007.1108

Patruno A., 2010b, ApJ, 722, 909

Pinto C., Middleton M. J., Fabian A. C., 2016, Nature, 533, 64

Pintore F. et al., 2016, MNRAS, 457, 2988

Plotkin R., Gallo E., Jonker P., 2013, ApJ, 773, 59

Pringle J. E., Rees M. J., 1972, A\&A, 21, 1

Psaltis D., Chakrabarty D., 1999, ApJ, 521, 332

Rana V. et al., 2016, ApJ, 821, 103

Rappaport S., Markert T., Li F. K., Clark G. W., Jernigan J. G., McClintock J. E., 1977, ApJL, 217, L29

Rees M. J., Begelman M. C., Blandford R. D., Phinney E. S., 1982, Nature, 295, 17

Remillard R., Levine A., 2008, ATel, 1853

Reynolds M., Reis R., Miller J., Cackett E., Degenaar N., 2014, MNRAS, 441, 3656

Ricci C., Beckmann V., Carmona A., Weidenspointner G., 2008, ATel, 1840

Romanova M. M., Ustyugova G. V., Koldoba A. V., Lovelace R. V. E., 2009, MNRAS, 399, 1802

Ross R., Fabian A., 2005, MNRAS, 358, 211

Rutledge R., Bildsten L., Brown E., Pavlov G., Zavlin V., 2002, ApJ, 577, 346

Sakano M., Warwick R., Decourchelle A., Wang Q., 2005, MNRAS, 357, 1211

Schulz N. S., Chakrabarty D., Marshall H. L., Canizares C. R., Lee J. C., Houck J., 2001, ApJ, 563, 941

Sidoli L., Paizis A., Mereghetti S., Götz D., Del Santo M., 2011, MNRAS, 415, 2373

Sleator C. C. et al., 2016, ApJ, 827, 134

Strohmayer T., Zhang W., Swank J., Smale A., Titarchuk L., Day C., Lee U., 1996, ApJL, 469, L9

Tomsick J., Yamaoka K., Corbel S., Kaaret P., Kalemci E., Migliari S., 2009, ApJL, 707, L87

Verner D., Ferland G., Korista K., Yakovlev D., 1996, ApJ, 465, 487

Vrtilek S. D., McClintock J. E., Seward F. D., Kahn S. M., Wargelin B. J., 1991, ApJS, 76, 1127 


\section{$12 \quad$ N. Degenaar et al.}

Wagner R. M., Starrfield S. G., Hjellming R. M., Howell S. B., Kreidl T. J., 1994, ApJL, 429, L25

Weng S. S., Zhang S. N., Yi S. X., Rong Y., Gao X. D., 2015, MNRAS, 450, 2915

Wijnands R., 2008. American Institute of Physics Conference Series, Vol. 1010, pp. 382-386

Wijnands R., van der Klis M., 1998, Nature, 394, 344

Wijnands R. et al., 2006, A\&A, 449, 1117

Wijnands R., Degenaar N., Armas Padilla M., Altamirano D., Cavecchi Y., Linares M., Bahramian A., Heinke C. O., 2015, MNRAS, 454, 1371

Wilkins D. R., Fabian A. C., 2012, MNRAS, 424, 1284

Wilms J., Allen A., McCray R., 2000, ApJ, 542, 914

Yang Q. X., Xie F. G., Yuan F., Zdziarski A. A., Gierliński M., Ho L. C., Yu Z., 2015, MNRAS, 447, 1692 